\title{
Dynamic interactions between lumbar intervertebral motion segments during forward bending and return
}

Breen Alexander ${ }^{1}$, Breen Alan $^{2}$

${ }^{\prime}$ Centre for Biomechanics Research, AECC University College, UK

${ }^{2}$ Faculty of Science and Technology, Bournemouth University, UK

\section{Introduction}

Continuous dynamic multi-segmental studies of lumbar motion have brought new depth to our understanding of the biomechanics of back pain and these are becoming more prevalent than static radiographic studies in research. They are needed for the clinical validation of both laboratory and FE modelling outputs that include motion (Jones and Wilcox 2008, Oxland 2016) and are necessary before in vivo studies of loading can be attempted during bending tasks. Our previous work showed that it may be feasible to do this by adding finite element models from MRI to kinematic information from fluoroscopy to estimate intervertebral loading during motion, thereby revealing the time points when stresses are maximal (Zanjani-Pour 2018). However, we also now know that the motion shared between vertebral segments is more variable and less repeatable during loaded than passive recumbent bending and also changes during motion (Breen, Hemming et al. 2019). This represents a challenge to attempts to compare individuals or populations or to establish normative values. This highlights the need to explore the nature of the interactions between motion segments during these bending tasks.

Previous studies have suggested that passive recumbent lumbar flexion presents greater unevenness of intervertebral motion sharing in patients with chronic, non-specific back pain (CNSLBP) than asymptomatic controls, but did not find a difference during loaded flexion or explore interactions between segments (Breen and Breen 2018, Breen, Mellor et al. 2018). Several studies have explored how angular motion is shared between segments of the lumbar spine at points during weight bearing flexion in both patients with back pain and healthy controls using either medical imaging or surface markers (Teyhen, Flynn et al. 2007, Ahmadi, Maroufi et al. 2009, Aiyangar, Zheng et al. 2015, Christe, Redhead et al. 2016, Gombatto, D'Arpa et al. 2017, Hemming, Sheeran et al. 2017, Papi, Bull et al. 2019). These found greater flexion ranges in the upper than lower lumbar spine in patients when compared to controls, however, no weight bearing studies have attempted to continuously measure the proportions of the flexion and return motion that is accepted by individual levels, or to describe the dynamic interactions between them during bending. This will be needed if we are to model contemporaneous kinematics and loading to estimate relative intersegmental stresses during bending motion. 
The purpose of this study was to assess the motion contributions of adjacent lumbar levels during an active weight bearing flexion and return protocol using quantitative fluoroscopy. Data were collected using a guiding motion platform to minimise behavioural variation and allow the greatest effects to be obtained from the morphology and muscular activity during the motion.

\section{Methods:}

\section{Participants}

Eight patients with chronic non-specific low back pain (CNSLBP), yet without any obvious mechanical disruption (for example surgery or spondylolisthesis) received fluoroscopic imaging during flexion and return motion. These were matched for age and sex to 8 healthy controls who in turn were extracted from a database of $>100$ asymptomatic individuals who had performed the same task. Asymptomatic participants were included if they were between 21 and 80 years old, had a selfreported body mass index of less than $30 \mathrm{~kg} . \mathrm{m}^{-2}$, were free of any back pain, had not experienced back pain that limited their normal activity for more than 1 day in the previous year, had no history of abdominal surgery or spondylolisthesis, had not received a medical radiation exposure of $>8 \mathrm{mSv}$ in the previous 2 years, and were not currently pregnant. Ethical approval was provided by the National Research Ethics Service (Bristol 10/H0106/65) and written Informed consent was obtained from all participants.

\section{Data collection}

The Quantitative Fluoroscopy (QF) systems and procedures have been detailed extensively in the literature (Breen and Breen 2017, Zanjani-Pour, Meakin et al. 2017, Breen, Mellor et al. 2018, du Rose, Breen et al. 2018, Breen, Hemming et al. 2019). However, in brief, participants undertook a standardised motion protocol during active weight-bearing flexion and return that reduces behavioural aspects of participant bending, guiding the participants speed and range of motion throughout their bend.

Participants were asked to fold their arms (left over right) out in front of them at chest height in a comfortable position while standing upright in a neutral posture, the arm rest of a guided motion control platform was then brought into position to meet the participants arms (See Figure 1). The participants were guided by the motion control platform at $6 \%$ to perform trunk flexion from upright standing to $60^{\circ}$ flexion, directly followed by guided return to a neutral standing position. During motion, the pelvis was constrained to reduce sacral translation but still allow some rotation of the hips. This was performed using a belt secured around the participants' hips and a bracing pad applied to the lower sacral segments (See Figure 1). Concurrently, fluoroscopic images were 
acquired using a Siemens Arcadis Avantic digital C-arm fluoroscope (Siemens GMBH) with the centre of rotation of the motion platform aligned with participants' L3/L4 intervertebral disc. During the bending protocol, fluoroscopic images were acquired at $15 \mathrm{~Hz}$ frame rate. These were transferred to a dedicated workstation where the vertebral body positions (L2, L3, L4, L5 and S1) were identified for each by a semi-automated tracking process written in Matlab (V2013, The Mathworks Inc.). This method has been previously validated and shown to have an accuracy in rotation measures of $0.52^{\circ}$ (Breen, Muggleton et al. 2006) and an inter- and intra-observer repeatability ranging from ICC 0.94$0.96\left(\right.$ SEM $0.23^{\circ}-0.61^{\circ}$ ) (du Rose A. and Breen 2016).

\section{Data analysis}

In order to investigate population differences in intersegmental spinal motion sharing metrics and intervertebral range of motion (IV-RoM) for each level, dynamic motion sharing of segments from L2-S1 were calculated throughout the bend and return.

Vertebral positions were established for each vertebra from L2-S1 and tracked throughout the bending sequence. To compare intervertebral motion sharing across and between populations, segmental motion profiles were normalised to a motion cycle as a percentage that clearly discriminated the outward (0-50\%) from the return phase (50-100\%). (See Figure 2).

Motion Sharing was calculated as the contribution of each motion pair as a percentage of the L2-S1 motion. Because segmental angular differences from the participants' starting positions are small at the beginning and end of participants' bending sequences, they are close to the precision limit of the QF Systems at these points ( 0.52 degrees). Therefore, contributions to motion sharing from points where the L2-S1 angle was less than $10 \%$ of the maximum L2-S1 RoM were truncated to remove the large relative contributions to errors (equivalent to data points at less than $5 \%$ and greater than $95 \%$ of the motion cycle) (Figure 3).

We calculated the average inequality of the motion share (Motion Sharing Inequality, MSI) and its standard deviation (Motion Sharing Variability, MSV) throughout the bend from the differences between maximum and minimum contributions throughout the flexion and return sequences. To do this, the range was calculated for each data point on the $\mathrm{x}$-axis. Then, MSI was calculated as the mean of all the ranges in the sequence and MSV as their standard deviation (Breen and Breen 2018). We also determined the average percentage contribution, for individual levels, across the motion (Average Motion Share, AvMS) and the standard deviation of each level's contribution across the motion (Motion Sharing per Level Variance, MS(L)V). Lastly, in order to compare against the literature, the percentage contribution at maximum bend (MS@max) was also computed. These 
were compared between groups and with a systematic review of spinal kinematics by Widmer et al. 2019 (Widmer, Fornaciari et al. 2019)

\section{Statistical analysis}

The normality of the data was calculated using the Shapiro Wilk test in SPSS (version 24, IBM Corp.). Independent t-tests were performed to test for differences between group data from a normally distributed dataset and Mann-Whitney $U$ was used for data that were not. Significance was set at $95 \%$.

Mean motion share contribution and $95 \%$ confidence interval $( \pm C \mid 95)$ values across all participants were computed at each $1 \%$ increment of the Motion Cycle of the controlled bending task for both the asymptomatic control and CNSLBP patient populations. Statistically significant differences between each level's contribution to motion was detected by the extent of overlap between the $\pm \mathrm{Cl} 95$ bands, i.e. the absence of $\pm \mathrm{Cl} 195$ band overlap indicated statistically significant differences.

\section{Results:}

Each participant group consisted of 5 males and 3 females matched for age and sex. Shapiro Wilk test for normality revealed that age, height and weight were likely to have come from a normally distributed data set, but BMI data were unlikely to be normally distributed. Furthermore, the Shapiro Wilk test found that motion metrics (range of motion and motion sharing within and between levels) were a mix of normal and non-normally distributed data depending on level. Therefore, for consistency all motion metrics were treated as non-parametric data. There were no significant differences between groups in terms of age, height, weight, or BMI (Table 1). However, the asymptomatic controls consistently gained higher ranges of intervertebral motion at all measured levels, although this was only significant at the L5-S1 level ( $p=0.012)$ (Figure 4 \& Table 2). The L2-S1 range of motion was also significantly less among the patient population $(p=0.046)$ (Figure 5 \& Table 2)

\section{Motion sharing inequality and variability}

Among controls, in initial flexion and the latter part of the return phase, there was a top down sharing of motion. However, at maximal bend the lumbar levels shared the motion more equally, with L5-S1 receiving the least (Figure 6). Among patients, similar contributions to motion can be seen during flexion, however, during return there was less symmetry of sharing, with L3-L4 continuing to receive more of the motion (Figure 7). 
Although different in appearance, the MSI and MSV values for patients and controls (Figures 6 and 7) were not significantly different. However, MS(L)V was significantly higher at L4-5 in the patients $(p=0.021)$. This lack of variation can be seen as a flatter curve, especially in the return phase of bending. (Figure 7).

\section{Individual level sharing}

Among controls, the average share of motion was highest at L2-L3 and lowest at L5-S1 and this tendency was greater with higher MSIs. Among patients, the average share of motion was highest at L3-L4 and lowest at L5-S1, the L5-S1 contribution being significantly different from the other levels throughout most of the bending protocol (as defined by the lack in of overlap of the $95 \% \mathrm{Cl}$ bands about the L5-S1 level with any other level in Figure 7)

\section{Comparison with the literature}

Few studies have examined intervertebral motion sharing during dynamic flexion and return tasks and none that can be compared directly. However, Widmer et al (2019) (Widmer, Fornaciari et al. 2019) recently presented a review of studies of lumbar kinematics and reported the segmental contributions to flexion from multiple studies. On the whole, two different types of segmental contribution profiles (spinal rhythms) were established. Type 1: A cranio-caudally decreasing contribution pattern, in protocols where total lumbar RoM was limited either by restricting the attempt or by starting the motion in a sitting position. Type 2: A cranio-caudally increasing contribution pattern with a slight drop at the L5-S1 segment, in protocols where lumbar RoM was unconstrained. Figure 8 and Figure 9, respectively, display these, with the control and patient data from the present study included for each level.

When calculating the average motion sharing during flexion and return (AvMS), it was noticed that the distribution of sharing was similar to Widmer's graph of limited flexion studies (Widmer, Fornaciari et al. 2019). That is, decreasing contributions per level between L2-L3 and L5-S1, with the exception of L3-L4 whose average contribution (AvMS) was greater in patients ( $p=0.046)$ (Figure 8 \& Table 2). This is consistent with L3-L4 and L4-L5 remaining in a relatively flexed position as demonstrated by the high contribution to L2-S1 angle during the return phase in Figure 7. This seems to characterise the difference in motion pattern between patients and controls.

In Figure 9, segmental contribution at maximum flexion for all studies, including the present one, shows a cranio-caudally increasing contribution, with a drop at the L5-S1 segment. This suggests that when participant range is standardised to $60^{\circ}$ of trunk bend, the lumbar segments (L2-S1) are flexed near to their maximal range. In the present study, which includes both patients and controls, 
the L5-S1 contribution at maximum was significantly lower in patients (Table 2) and significantly less than all other levels (Figure 7).

\section{Discussion:}

There were consistent but non-significant differences between patient and control motion sharing patterns. This lack of significance may be due to the range of L2-S1 motion of patients' spines being significantly less, particularly at the lower levels. The results also illustrate the effects of loading and muscle activity on the differences between lumbar flexion and return motion in controls and patients with CNSLBP. Widmer et al (2019) considered that contributions to flexion motion may be RoM dependent and this is consistent with our findings, where patients had lower L2-S1 RoM $(p=0.046)$ and a lower contribution at maximum bend at L5-S1 $(p=0.046)$.

Our previous studies of passive recumbent proportional motion did not dis-aggregate intervertebral levels, but unlike this study, did find MSI to be significantly higher in patients (Breen and Breen 2018, Breen, Mellor et al. 2018). These differences may be due to any combination of contributions from behavioural influence on bending strategy, involuntary muscle activity and changes in passive tissue restraint. For example, the increased variability of motion sharing in patients $(M S(L) V$ at $L 4-5$, $p=0.021$ ) may be consistent with the work of Du Rose et al (du Rose, Breen et al. 2018), who measured local and global lumbar SEMG activity during bending in controls and found that it correlated negatively with MSV. Considered in relation to patients, this may suggest a guarding effect. This present study did not include muscle oxygenation or electrical activity, which could shed considerable light on these issues.

A further finding was that whether in patients or controls, contributions to motion change continuously during bending. Although fairly consistent in groups, this makes static measurement of IV-RoM of limited use as it is dependent on the phase of flexion as well as the restraint of the segment.

Our finding that motion contributions change dramatically throughout the bend and seem to be RoM dependent are consistent with the findings of the review by Widmer et al (2019). Therefore, the significant reduction in patients' lumbar range of motion may be contributing to the significant differences between population motion sharing characteristics. It may also be true that motion sharing is dependent on the global position at which the participant starts their motion. This was not investigated but highlights the need to standardise data collection protocols and only include those which adhere to them in comparing studies. 
The dynamic interactions between lumbar intervertebral motion segments during weight bearing flexion and return were found to be different in patients with CNSLBP compared to healthy controls. However, although global motion of participants in both groups were $60^{\circ}, \mathrm{L} 2-\mathrm{S} 1$ maximum range was lower in patients, while individual level contributions changed during the motion and seem to be RoM dependent. Therefore, it is unsurprising that only L5-S1 was significantly different between groups in terms of motion sharing metrics. However, there also appears to be less variability in the motion contributions of different levels in patients, although these were not significant in these small populations. This lower variance in patients, particularly during return from full flexion, may be related to increased muscle contraction. Therefore, muscle workload needs to be verified and/or explained by further studies, with larger populations. These could include muscle electrical activity and oxygenation alongside kinematics and loading as well as comparisons with passive recumbent protocols within which muscle activity and loading are likely minimal.

\section{References}

Ahmadi, A., N. Maroufi, H. Behtash, H. Zekavat and M. Parnianour (2009). "Kinematic analysis of dynamic lumbar motion in patients with lumbar segmental instability using digital videofluoroscopy." European Spine Journal 18: 1677-1685.

Aiyangar, A., L. Zheng, W. Anderst and X. Zhang (2015). "Apportionment of lumbar L2-S1 rotation across individual motion segments during a dynamic lifting task." Journal of Biomechanics 48(13): 3709-3715.

Breen, A. and Ax. Breen (2018). "Uneven intervertebral motion sharing is related to disc degeneration and is greater in patients with chronic, non-specific low back pain: an in vivo, crosssectional cohort comparison of intervertebral dynamics using quantitative fluoroscopy." Eur Spine J 27(1): 145-153.

Breen, A., R. Hemming, F. Mellor and A. Breen (2019). "Intrasubject repeatability of in vivo intervertebral motion parameters using quantitative fluoroscopy." Eur Spine J 28(2): 450-460.

Breen, Ax., F. Mellor and A. Breen (2018). "Aberrant intervertebral motion in patients with treatment-resistant nonspecific low back pain: a retropective cohort study and control comparison." European Spine Journal 27(11): 2831-2839.

Christe, G., L. Redhead, T. Legrand, B. M. Jolles and J. Favre (2016). "Multi-segment analysis of spinal kinematics during sit-to-stand in patients with chronic low back pain." Journal of biomechanics 49(10): 2060-2067. 
du Rose, A., Ax. Breen and A. Breen (2018). "Relationships between muscle electrical activity and the control of inter-vertebral motion during a forward bending task." Journal of Electromyography and Kinesiology 43: 48-54.

du Rose A. and A. Breen (2016). "Relationships between lumbar inter-vertebral motion and lordosis in healthy adult males: a cross sectional cohort study." BMC Musculoskeletal Disorders 17(121).

Gombatto, S. P., N. D'Arpa, S. Landerholm, C. Mateo, R. O'Connor, J. Tokunaga, L. J. J. M. S. Tuttle and Practice (2017). "Differences in kinematics of the lumbar spine and lower extremities between people with and without low back pain during the down phase of a pick up task, an observational study." 28: 25-31.

Hemming, R., L. Sheeran, R. van Deursen and V. Sparkes (2017). "Non-specific chronic low back pain: differences in spinal kinematics in subgroups during functional tasks." European Spine Journal. Jones, A. C. and R. K. Wilcox (2008). "Finite element analysis of the spine: Towards a framework of verification, validation and sensitivity analysis." Medical Engineering \& Physics 30(10): 1287-1304.

Oxland, T. R. (2016). "Fundamental biomechanics of the spine - What we have learned in the past 25 years and future directions." Journal of Biomechanics 49(6): 817-832.

Papi, E., A. M. Bull, A. H. J. G. McGregor and posture (2019). "Spinal segments do not move together predictably during daily activities." 67: 277-283.

Teyhen, D. S., T. W. Flynn, J. D. Childs, T. R. Kuklo, M. K. Rosner, D. W. Polly and L. D. Abraham (2007). "Fluoroscopic Video to Identify Aberrant Lumbar Motion" Spine 32(7): E220-E229.

Widmer, J., P. Fornaciari, M. Senteler, T. Roth, J. G. Snedeker and M. Farshad (2019). "Kinematics of the Spine Under Healthy and Degenerative Conditions: A Systematic Review." Annals of Biomedical Engineering: 1-32.

Zanjani-Pour, S., Meakin, J,R,, Breen, Ax., Breen A. (2018). "Estimation of in vivo inter-vertebral loading during motion using fluoroscopic and magnetic resonance image informed finite element models." Journal of Biomechanics 70: 134-139. 